\title{
Study of the collection solid angle of doubly curved crystals
}

\author{
Sa J Pestehe', Gh Askari Germi ${ }^{2}$, and A Rastkar Ebrahimzadeh ${ }^{2}$ \\ 1. Department of Physics, University of Tabriz, Tabriz, Iran \\ 2. Department of Science, Azerbaijan Shahid Madani University, Tabriz, Iran
}

E-mail: sjpest@tabrizu.ac.ir

(Received 12 October 2018 ; in final form 07 August 2019)

\begin{abstract}
One approach to obtain a maximum solid angle is the use of curved crystals. Therefore, in order to make these crystals useful in X-ray spectrometry, it is necessary to design them in such a way that they have high solid angle and reflectivity. In this paper, a nearly exact general equation for calculating the solid angle and area factor on the surface of several curved crystal geometries is extracted and compared with the previous results. Wittry and Sun's shortcut method is applied to calculate the solid angle; also, its trial and error method is employed to maximize the solid angle and the introduced exact point-focusing crystal geometry is reviewed; it is shown that for some crystal geometries, they are not responsive. By writing an algorithm for calculating the solid angle and the area factor for all of the crystal geometries, we show that they are in agreement with the results of the analytical method.
\end{abstract}

Keywords: collection solid angle, toroidally bent X-ray diffractors, effective scattering area on the crystal surface, point-focusing crystal geometry

For full article, refer to the Persian section 